\title{
DÉSIR DE MORT ET PUISSANCE ABSOLUE DE CHARLES VIII A HENRI IV
}

L'État de la première modernité - la proposition n'a rien d'original - est fondé sur le sacré, sur une union de la nature et de la surnature en la personne d'un roi rituellement sacré et investi d'une Majestas dans la prise en charge même de l'office royal ${ }^{1}$. Le souverain est puissance et cette puissance est de Dieu. Le regard extraordinaire du roi et le toucher des écrouelles sont les signes les plus marquants de cette situation intermédiaire entre sphère humaine et sphère divine, de cette plénitude unique en un homme désigné par Dieu pour régir son peuple selon sa Loi et l'aider à se préparer à l'autre vie. Pour les hommes du premier $x v^{e}$ siècle, le corps royal d'un François $I^{\mathrm{er}}$, dans sa beauté qui ne fait que refléter une âme ornée de toutes les vertus, témoigne aussi d'une élection divine qui, individuellement conférée, transcende le rituel même du sacre. Et le corps du royaume, par l'exaltation d'une totale osmose à la volonté du vicaire de Dieu, est destiné, dans le système de représentation, à souligner l'évidence d'une perfection de la construction étatique découlant de la perfection sacrale du monarque - monarque prophétiquement désigné pour réaliser l'unité du monde dans la foi du Christ.

Même si, pour citer A. Dupront, «le cérémonial du sacre ne va pas jusqu'à consacrer le souverain dans la toute-puissance du règne [...] car Dieu est un et le règne appartient à son entièreté seul ${ }^{2}$, il n'en est pas moins vrai que l'idéologie du pouvoir renaissant tire sa force de son triomphalisme, qui lui permet d'affirmer que le roi, corporalis deus, est vie parce qu'unité unifiante des multiplicités, principe actif de vie sans lequel le royaume ne serait que chaos et ténèbres, le corps politique que mort et pourriture. La métaphore du soleil est déjà en cuvre, légitimant

1. Sur ce point, Alphonse DuProNT, « Sacre, autorité, pouvoir : profil d'anthropologie historique ", in Le Sacre des rois. Actes du Colloque international d'histoire sur les sacre et couronnement royaux, Reims, 1975, Paris, Les Belles Lettres, 1985, p. 315-350.

2. Ibid., p. 323.

Revue de synthèse : IV' S. Nos 3-4, juil.-déc. 1991. 
l'absoluité de l'autorité et coexistant avec l'identification toujours plus implicite du monarque terrien au Christ éternel. Là sont les données massivement apparentes d'un système qui se déploie avant tout dans des répétitions rituelles, représentations et croyances et qui fonctionne par l'artifice même de ce jeu de rituels, de représentations et de croyances. Le pouvoir des derniers Valois - c'est sa faille cachée - est d'abord un imaginaire, imaginaire de la vie en un homme vivant pour tous les hommes. Il est ainsi primordialement un discours que la symbolique même de l'Hercule Gaulois rend perceptible. C'est par le langage que le roi se fait obéir de ses sujets, par le mythe, et il est évident que la fragilité de l'architecture étatique renaissante tient à ce que tout est construit moins sur une réalité du pouvoir absolu que sur le pouvoir rhétorique des images et des rituels.

Toutefois le sacré n'est pas que langage et symbole, idéologie et institution. Premièrement, parce que le sacre apparaît autant comme une sanction d'une "grâce " préexistante que comme une consécration, à travers la conceptualisation de la continuité monarchique et du double corps ${ }^{3}$. Et Y.-M. Bercé de constater, dans cette perspective, que «le roi ne peut s'empêcher d'être roi; sa légitimité ne dépend pas de l'histoire et de ses contingences, elle résulte des décrets du Ciel... ${ }^{4}$. Secondement et essentiellement, parce que le sacré est aussi un désir, un art de créer une représentation qui définisse le pouvoir sur un mode liminal ou extrême par rapport à la durée humaine. C'est-à-dire que, pour percevoir le plein sens de la monarchie de la première modernité, il faut déporter l'analyse vers l'étude de la relation à la mort qui pourrait être celle du roi, relation d'autant plus fondamentale que le $\mathrm{xvI}^{\mathrm{e}}$ siècle, quelques décennies après le décès d'Henri II au cours d'un jeu de violence, se clôt précisément par deux fois par la mort régicide.

Par-delà les faits simples et en apparence seulement antinomiques que le pouvoir est pouvoir de donner la mort - « la mort est son droit ultime et aussi bien ce dont il protège les autres $"{ }^{5}$ - et que le roi vivant demeure à l'écart des cérémonies qui solennisent la mort de son prédécesseur dans la dignité royale, une première question peut être posée : dans une civilisation remplie par le va-et-vient de la mort, dans laquelle le Prince s'énonce distributeur de vie et de mort, le désir de mort n'est-il pas une des forces qui assure le fonctionnement même de l'idéologie sacrale

3. Michel Devèze, « La doctrine d'Olivier Martin sur la monarchie française de droit divin ", in op. cit. supra n. 1, p. 243-248.

4. Yves-Marie Berce, Le Roi caché. Sauveurs et imposteurs, mythes politiques populaires dans l'Europe moderne, Paris, Fayard, 1989, p. 380.

5. Marc AugE, Pouvoirs de vie, pouvoirs de mort. Introduction à une anthropologie de la répression, Paris, Flammarion, 1977, p. 122. 
de l'État? Le roi n'est-il pas certifié et reconnu l'homme commissaire de Dieu sur terre parce que, pour tous, il se voue personnellement à frôler et à assumer la mort, dans une tension sacrificielle dont la finalité serait d'authentifier la coexistence en lui de la nature et de la surnature, dans la forme de maîtrise de la mort qu'est le mépris même de celle-ci ?

Un fantasme qui court dans la société huguenote sur la fin de 1559 ou au tout début de 1560 , quand se délite ou se défait la construction idéologique du premier absolutisme, n'est-il pas significatif de la valeur axiale de cette intimité du Prince avec la mort, dans un retournement qui tend à le diaboliser absolument? Un rapport s'établit entre la crise du pouvoir et une image inversement sacrificielle du roi : c'est l'essor d'une rumeur prétendant que dans le royaume circulaient "certains personnages " qui avaient reçu commission de se saisir des « plus beaux et des plus sains enfans qu'on pourroit trouver, de l'âge de 4 jusques à 6 ans ". Le jeune François II devait être baigné dans leur sang afin que soit régénérée une vie qu'on savait fragile. Sa survie allait être redevable de la vie des innocents, de ceux qui sur terre sont des figures de la pureté christique, et déjà, selon l'Histoire ecclésiastique, "se trouva que plusieurs incognus avoient este ça et là en quelques villages, demandans es maisons et escrivans en quelque papier le nombre, l'age et le nom des enfans ${ }^{6}$. Le corps du roi enduit de sang allait revivre de la vie sacrifiée de ceux qu'il est de son devoir même de protéger, dans une manière d'onction monstrueuse.

Une face cachée ou une configuration souterraine du pouvoir s'éclaire ici indirectement. Si l'anti-roi est, dans l'imaginaire collectif de la veille des guerres de religion, un roi antéchristique qui sacrifie les siens dans un rituel sanglant annonçant déjà les représentations ligueuses du « meurtre inhumain et barbare " de Blois, n'est-ce pas parce que la donnée brute du pouvoir est au contraire le sacrifice continué du roi, à la fois désir même du pouvoir et désir mimétique de ceux qu'il régit? Ce désir impose l'amour, amour du roi pour des sujets qui s'unissent en son obéissance, amour des sujets pour leur roi, à commencer la noblesse « tousiours estrainte et affectionnée au prince, et preste à le servir en toutes affaires, et exposer les biens et le corps pour la défense du royaume et pour le service du roi " ${ }^{7}$. La tension de sacrifice n'est-elle pas l'au-delà de la royauté rituellement sacrée du $\mathrm{Xvi}^{\mathrm{e}}$ siècle, ce par quoi sans cesse elle se recompose

6. Théodore de BĖZ, Histoire ecclésiastique des églises réformées au Royaume de France..., à Anvers, de l'imprimerie de Jean Remy, 1580, t. I, p. 244.

7. Claude de Seyssel, La Grand' Monarchie de France, A Paris, Par Galiot du Pré, 1558, p. 37, qui ajoute : "Car aussi la raison le veult, pourtant qu'à la verité ce sont ceux qui ont faict les haulx et louables faictz dont le royaume a esté du commencement estably, et après augmenté et conservé : et de tous temps l'ont defendu, et sont tousiours prestz à mettre et exposer leurs vies pour la defense d'iceluy : et si ont plus d'amour et de reverence au prince que nul des autres... " 
ou se relégitime comme ordre de Dieu avant que ne s'impose au $\mathrm{XvII}^{\mathrm{e}}$ siècle une monarchie fondée primordialement sur la Raison?

Le cauchemar prophétique qui surprend le sommeil de Montluc la veille de la mort du roi Henri II et qui le traumatise au point de le faire pleurer à son réveil témoigne de cette relation étroite qui structure l'image du roi. Pour l'auteur des Commentaires, Henri II est le roi aimé entre tous les rois qu'il a servis, celui qui n'aurait pas dû mourir parce qu'il était le seul à pouvoir empêcher le royaume d'imploser en des guerres civiles; Henri II dont pourtant la mort est rêvée dans une anticipation qui semble relever d'une intense angoisse.

L'ambiguïté est certaine. D'une part, on peut penser que la préscience de la mort du roi procède d'une subjectivité saisie par l'enchaînement prophétique d'un monde qui bascule toujours plus dans la désunion religieuse et qui ne peut que subir toujours plus le châtiment divin - dont la disparition d'Henri II s'avérera un des signes capitaux. Trois jours auparavant, dans une conversation avec Antoine de Bourbon, Montluc avait déjà eu des paroles prémonitoires :

« que j'avais tout jamais ouy dire que, quand un homme pense estre hors de ses affaires et qu'il ne songe qu'à se donner du bon temps, que c'est alors qu'il luy viennent les plus grands mal'heurs, et que je craignois la sortie de ce tournoy..."

D'autre part, cette mort n'est-elle pas à penser comme le désir de ceux qui, comme Montluc, ne cessent de se raccrocher aux valeurs anciennes du temps des guerres d'Italie, avant le « malheur » de la signature du traité de Cateau-Cambrésis? Ne représente-t-elle pas cette essence du pouvoir qu'est la mort sacrificielle, comme pour tenter, dans la fiction du temps du rêve, de l'isoler pour mieux laisser croire encore en elle ? Le rêve n'est-il pas la réalisation d'un désir au seuil de la grande crise de l'État, et le sang qui coule sur le visage du monarque n'est-il pas l'idéalité absolue de la royauté absolue que Montluc sent se dissoudre?

« Et la nuit propre venant au jour du tournoy, à mon premier sommeil, je songeay que je voyois le Roy assis sur une chaire, ayant le visage tout couvert de gouttes de sang; et me sembloit que ce fust tout ainsi que l'on peint Jesus Christ, quand les Juifs luy mirent la couronne, et qu'il tenoit ses mains joinctes ${ }^{8}$.

L'important est ici que, dans la violence et dans l'image de mort, le roi rejoint le Rédempteur de l'humanité, que sa mort s'impose comme sacri-

8. Blaise de Montluc, Commentaires, 1521-1576, préf. par Jean Giono, éd. critique établie par Paul Courteault, Paris, Gallimard, 1964, p. 464-465. 
fice et que le désir, dans le temps privilégié qui met en action le théâtre privé du rêve, est désir de fixer une vérité du pouvoir, que Henri II, en négociant la paix avec l'Espagne, a soudain effacée, gommée, rendue vaine et passive. Henri II est le roi qui pour la noblesse a perdu son double ontologique, ou encore qui a rejeté sa mort potentielle et que le rêve restaure, en un désir effrayant, en plénitude ${ }^{9}$; ce, avant que dans le jeu dangereux du tournoi l'histoire même ne le recompose en un événement réel de rupture. La vraie mort du roi va rendre le désir réalité. Il suffit de remonter dans l'histoire pour se rendre compte de ce que le rêve du capitaine, dans une surcharge dramatique, tentait de redonner vie à des images structurelles.

Car un des temps du roi, au cours du premier $x^{i}{ }^{e}$ siècle, est un temps d'approche de la mort, d'un défi constamment lancé à la mort et aux forces de mort à partir duquel son identité sacrée s'amplifie ou se dilate à l'extrême en laissant reconnaître l'extraordinaire d'une virtus divine agissant en lui. Charles VIII, dans la mythologie qui se constitue lors du voyage d'Italie, est autant le roi libérateur que les prophéties ont annoncé que le roi qui affronte sacralement la mort, se laissant volontairement comme aller jusqu'à ses extrêmes frontières.

Dans la vision commune en effet, après la conquête du royaume de Naples, le repli de l'armée se transforme en une geste prodigieuse dans laquelle le roi est le centre de tous les regards et de toutes les actions. La bataille de Fornoue devient sous la plume de Jehan de Bourdigné une œuvre de justice voulue par Dieu, destinée à punir par la «férocité gallique " la déloyauté et la trahison des «parjures et mal fidelles Italliens $"{ }^{10}$. Elle a lieu lorsque l'armée française, harcelée, fatiguée et menacée, semble devoir être défaite, entourée par les pièges et les ruses de l'ennemi. Entre Charles VIII et les ligués, le combat se fait combat de Dieu contre les passions humaines, combat contre la Faute, dans un élan collectif qui procède d'abord de l'engagement sacrificiel du roi de France. Le sang italien qui va couler à flot sera le sang du péché. La violence de Charles VIII fait la violence de ses guerriers et c'est parce que lui-même ignore la mort que tous ceux qui le servent sont représentés se lançant au combat en ignorant la mort. La volonté sacrificielle royale implique la

9. Sur ce rapport du double à la mort, Jean BAUDRILLARD, L'Échange symbolique et la mort, Paris, Gallimard, 1976, p. 216-222 et René GIRARD, La Violence et le sacré, Paris, Grasset, 1972.

10. Jehan de Bourdigne, Chroniques d'Anjou et du Maine, Angers, de Quatrebarbes éd, 1842, p. 268 : « ... Et ainsi, comme dit le poete Fauste, les victoires et bellicqueux faictz du preux roy Charles sont stigmates imprimez au fronc et entendement des Italliens qui jamais n'en tomberont. " 
violence de toute une armée prête à mourir pour son roi, pour la « patrie " et donc pour Dieu.

De plus, dans le déchaînement simultané des hommes et des éléments, unis dans une puissance inouie, la bataille s'apparente au Jugement dernier, dont les Français deviennent aux côtés de leur roi les acteurs désignés par Dieu pour châtier ceux qui se sont séparés de Lui :

« durant la chasse que les François faisoient après eulx fuyans, tonnoirres, choruscations, foudres, esclers, gresles, ventz et oraiges sourvindrent, en telle tempeste et tourment qu'il sembloit que les catharactes du ciel fussent ouvertes, et que le monde deust finir..."

Le roi, tel l'ange des derniers Temps, est à la tête des combattants français dont le nombre est rapporté dérisoirement faible face à leurs adversaires. Sa hardiesse et sa vaillance l'isolent, non pas seulement parce que de sa propre main il tue deux des gentilhommes vénitiens les plus notables et en met en fuite trois autres ", mais surtout parce qu'elles le révèlent porté par un mouvement d'absolue violence, ignorante de tout danger et pourtant victorieuse de toutes les agressions.

La violence est le sacré. Elle re-produit le sacré, le fait monter dans l'ordre fini du temps humain à l'occasion de l'immense désordre qu'elle sécrète, du chaos morbide qu'elle instaure. Charles VIII ne regarde que l'ennemi et, dans le combat, rien n'importe plus pour lui que de dispenser sa force sur les corps des hommes qui se sont levés contre son armée. Sur son cheval noir, il devient lumière, portant sur son armure une « jacquette " de couleur blanche et violette, parsemée de petites croix de Jérusalem "faictes de fines broderies et enrichies d'orfœuvrerie " ${ }^{12}$. La représentation que transcrit dans ses Mémoires le chevalier Guillaume de Villeneuve est centrée sur une totale tension, à la fois émulatrice et révélatrice :

« car c'estoit celluy qui tousjours eust la face droit à ses ennemys, l'espée au poing, la bouche plaine de bonnes et vertueuses paroles à ses gens. Et le fait de mesme le cueur plus gros que le corps avecques la fierté de ung lyon, tant que la bataille dura, et après la victoire doulx et begnin comme ung ange, recognoissant la grant grâce que Dieu luy avoir faite $"{ }^{13}$.

L'image alors se dédouble : le roi Charles, après avoir été un nouveau David affrontant Goliath, s'authentifie comme un second Charlemagne,

11. Pierre de Bourdeille, seigneur de Brantome, Vies des hommes illustres et capitaines françois, t. I, in Euvres completes, Paris, Foucault, 1823, vol. 2, p. 189.

12. Ibid., p. 8.

13. Mémoires de Guillaume de Villeneuve, in Nouvelle collection des mémoires..., éd. Michaud-Poujoulat, t. IV, Paris, 1866, p. 383-384. 
car ce n'est pas uniquement pour la victoire et sa gloire personnelle qu'il combat ; en effet, s'il ignore le danger, c'est aussi parce qu'il est au milieu des siens, qu'il n'a de cesse de venir secourir lorsque "besoin en estoit » et au péril de sa propre vie. Il s'expose continuellement à la mort, prêt au sacrifice pour préserver sa noblesse au point d'être blessé et de se retrouver seul au milieu des ennemis. Tout d'abord, comme refoulant hors de lui-même, dans la pratique même de la violence, la puissance sacrale qui s'incarne en sa personne, il se fait pure humanité.

Il est un homme parmi les siens, un homme qui peut, voire désire mourir comme tous les siens pour l'unique gloire de Dieu et de la France éternelle. Il fait offrande de lui-même. Ce n'est que dans un second temps que la bataille le désigne et consacre roi vertueux, dépositaire absolu de la force sublimante qu'est la virtus dei, et Commynes ne peut s'empêcher de se remémorer l'extraordinaire d'une transfiguration physique, d'une autre incarnation en lui; il se rappelle que le roi, pourtant de petite taille et de complexion fragile, normalement empreint de timidité, subitement paraissait « tout autre que sa nature ne portoit " tant son cheval « le monstoit grand et avoit la parole audacieuse, et sembloit bien (et m'en souvient) que frère Hieronyme m'avoit dit vray, quand il me dit que Dieu le conduisoit par la main... ${ }^{14}$. Un autre être surgit en lui, qui est l'Autre.

La bataille de Fornoue, res mirabilis, si elle développe dans son déroulement un dépassement des contingences humaines dans le corps du roi, nomme ainsi ce dernier roi d'amour, " naturel maistre " de ses bons et loyaux sujets qui n'hésitent pas à venir affronter les adversaires pour le secourir, triomphateur de la mort qu'il domine lui-même dans le merveilleux d'un corps à corps de tous les instants et dans le soutien qu'il apporte à ceux qui luttent à ses côtés.

C'est-à-dire que la bataille, œuvre de Dieu à travers l'acte royal de sacrifice, enferme les combattants français dans un temps et un espace sacrés; elle les fait participants d'une geste merveilleuse, d'un mystère au cours duquel l'humain rejoint le Divin, ou plutôt le Divin rejoint l'humain. Un pouvoir sacré trouve alors sa totale cohérence dans le mépris mystiquement régénérant de la mort qui a été celui du roi et qui a été si intense que Charles VIII a failli se laisser surprendre sur la fin de la bataille : il est demeuré isolé avec son valet de chambre Antoine des Ambus, tandis que les neuf preux qui portaient la même armure que lui pour mieux tromper l'ennemi s'étaient laissés prendre au jeu de sa propre violence au point d'oublier qu'ils devaient le protéger et de se retrouver « un peu espars » :

14. Philippe de Commynes, Mémoires, in op. cit. supra n. 13, p. 207. 
« Une bande petite, de quelques hommes-d'armes desrompus, qui venoient au long de la grève qu'ils voyoient toute nette de gens, vindrent assaillir le Roy et ce valet-de-chambre; ledit seigneur avoit le meilleur cheval du monde, et se remüoit et se deffendoit; et arriva sur l'heure quelque nombre de ses autres gens, qui n'estoient guères loin de luy, et lors se mirent les Italiens à fuir $" 15$.

Et comme si la victoire re-créait ou re-consolidait une identité collective qui serait sacrale, naturellement confirmée dans l'acte ontologique d'union des sujets à un Prince épousant le dessein d'une offrande de soi à la gloire de Dieu, le lendemain c'est moins l'armée du roi que l'armée de Dieu qui échappe aux troupes liguées quand, après le délogement, l'absence de guide est perçue.

" Notez qu'il ne falloit point de guide; car Dieu seul avoit guidé la compaignie au venir, et ensuivant ce que m'avoir dit frère Hieronyme, il nous vouloit encores conduire au retour : car il n'estoit point à croire qu'un tel Roy chevauchast de nuict sans guide là où il ne pouvoit assez finer. Encores nous monstra nostre seigneur plus grand signe de nous vouloir preserver; car les ennemis ne s'apperçurent point de nostre partement qu'il ne fût midy... " ${ }^{16}$.

La guerre, surtout dans cette mêlée qui, dans les représentations, simultanément pour le roi et ses guerriers, reproduisait le combat paradigmatique de David contre Goliath, du petit contre le grand, de la foi contre la force, semble avoir été l'occasion d'un basculement de tous dans un ordre unitaire parce que sublimé, action initiatique assurant d'une unité de tous dans la Vérité de l'Éternitë. Elle est l'Esprit contre la matière, un acte de perpétuation de l'Acte créateur. Elle réobjective triomphalement ce qu'Alphonse Dupront a nommé,

" dans la certitude de l'organicité de la création divine, l'ambition métaphysique du sacre et pourquoi celui-ci apparait, au regard anthropologique, comme l'un de ces actes insignes où le groupe social concentre à l'extrême ses puissances vitales afin d'atteindre à une transcendance manifestée et vivre une promesse d'éternité ${ }^{17}$.

La violence sacrificielle ne semble-t-elle pas alors conditionnelle de l'étonnant équilibre de la royauté renaissante, de cette cohésion qui

15. Ibid., p. 211-212. Et à l'issue du combat au soir, le roi maintient ce lien privilégié avec ceux qui ont combattu pour lui contre la mort : " Je vis le Roy en sa chambre où il y avoit des gens blessez, comme le seneschal de lion et autres qu'il faisoit habiller... "

16. Ibid., p. 213.

17. A. DUPRONT, art. cit. supra n. 1, p. 327. 
frappa les contemporains entre un pouvoir dont le premier objet était de « faire croire " en son absoluité et un peuple hanté par la quête du salut?

Dans l'impulsion de ces premières images, tous les rois se veulent des rois combattants qui, emmenant leur noblesse vers l'Italie dans la quête de l'honneur, façonnent la volonté de Dieu. L'engagement de l'armée dans une lutte décisive est appréhendé comme un temps privilégié parce qu'autorisant un surgissement de Dieu : c'est Louis XII à Agnadel, le 14 mai 1509 , qui, loin de ressentir le moindre doute ou effroi devant la violence qui va éclater et ne pourra qu'être terrible, «feust tout ce jour [...] fort joyeux et de bon visage, allant toujours durant la bataille de bande en bande et de quartier en quartier, donnant bon courage à ses gens, en leur disant tout plain de belles parolles $"{ }^{18}$. Seul l'épuisement physique l'empêcha de continuer à frapper les corps ennemis ${ }^{19}$.

Mais, peut-être absolue nécessité du pouvoir absolu parce qu'instant de rencontre avec l'Absolu, le face-à-face royal avec la mort n'intervient pas uniquement dans l'instant de la guerre ouverte. On le note ainsi lorsque le roi de France séjourne à Asti, et qu'un soir, alors que toute l'armée est au repos, un valet d'étable, "par defaut de mettre de l'eau en son vin, s'endormit en sa litière sans souffler sa chandelle ». Peu après, sur les onze heures de la nuit, la maison prend feu; les cloches sonnent le tocsin, "dont plus de vingt mille hommes de la ville, au son du feu, furent soudainement par les rues, et à tous grands cris coururent où étoit le feu ". Cette mobilisation des habitants d'Asti, selon Jean d'Auton, déclenche une mobilisation spécifique de l'armée royale, « et ainsi le cas le requerroit assez, vu le son tumultuaire, l'heure intempestive, la multitude des etrangers, et la sûreté non fiable du pays, qui là à chacun étoit en vue ". Tout d'abord le roi est décrit se levant, " habillé légèrement ", et la hâche au poing. Il descend en compagnie d'un valet de chambre, dans la cour de son logis, puis avec ses gardes se dirige vers la rue d'où venait «le bruit». C'est donc lui qui affronte le premier physiquement le danger potentiel de la ville. Puis il est rejoint par tous les Français qui sortent de leurs logis à la crainte que " en la ville y avoit quelque hutin " [querelle]. Les uns, comme leur roi, ont la hâche à la main, les autres portent une hallebarde ou une pique. En moins d'un quart d'heure, ils sont six mille autour de Louis XII, illuminés par les lumières de douze cents torches, qui marchent jusqu'à l'emplacement du sinistre. Mais le chroniqueur, Jean d'Auton, de préciser que « en ce point s'en alla le roi jusques au lieu où estoit le bruit, comme celui qui hardiment se vouloit trouver où besoin

18. Robert de LA MARCK, Histoires des choses mémorables advenues du reigne de Louis XII et François $F^{\prime}$ en France, Italie, Allemagne et es Pays-Bas depuis l'an 1499 jusques en l'an 1521, in op. cit. supra n. 13, t. V, p. 13-14.

19. Bernard QUillet, Louis XII, Paris, Fayard, 1986, p. 390. 
estoit $"{ }^{20}$. Le roi est donc le personnage qui, dans la société terrestre, fait front au chaos, au péril, au devenir, à la mort, celui qui accepte totalement son humanité pour être ensuite reconnu au-delà de l'humanité. La maîtrise de l'ordre humain est violence parce que la violence autorise l'authenticité d'une révélation. La sacralité est action, désir de combattre.

Et ce désir ne quitte pas le souverain, même quand l'instant ultime semble s'approcher. Une obsession qui signifie cette dimension primaire du pouvoir qu'est la lutte contre la mort appréhendée toujours et jusqu'au bout de la vie comme un corps à corps. En avril 1505, Louis XII tombe malade. Tout le royaume prie et processionne pour lui. Il semble bientôt perdu, et c'est alors que comme pour se préparer à un affrontement, il fait part à ceux qui l'entourent d'une exigence :

" or fut le roi, durant cette maladie, par defaut de repos, tant affoibli, que ses spirituels sensitifs entrèrent en rêverie, et, après divers propos, demanda Madame Claude, sa fille, laquelle fut présentée par Madame de Tournon, sa gouvernante; puis voulut avoir son épée et une javeline dont il se souvint lors : pour lui complaire, lui fut baillé, au lieu de ce, quelque bâton, lequel voulut bailler à Madame Claude, disant que nul autre qu'elle, s'il ne vouloit incontinent mourir, y touchât ${ }^{2 !}$.

L'anecdote ne fixe-t-elle pas la dimension primairement violente du sacré royal, qui doit comme se poursuivre dans le théâtre des derniers instants?

Une manière d'apogée de cette représentation épiphanique de la royauté est bien sûr atteinte avec François $I^{\text {er, }}$ A.-M. Lecoq l'a montré magistralement. Marignan, sous la plume des narrateurs, devient le jour de la Sainte-Croix une victoire de la Croix et le vainqueur est consacré comme un nouveau Constantin ${ }^{22}$. Le roì, marchant avec les bandes noires, est au cceur d'une mêlée d'autant plus confuse que les Suisses "portoient pour leur signal la croix blanche, aussi bien que les François, ne portans pour différence sinon une clef de drap blanc chacun en

20. Jean d'Auton, Chroniques, Paris, P. L. Jacob éd., 1834, t. II, p. 184. Par la suite, la gouvernante ayant touché au bâton, il faut faire croire au roi qu'elle est morte, la lui faire apparaître venant du paradis...

21. Ibid., t. III, p. 120.

22. Mais, dés le 28 août 1514 , le pressentiment d'un événement extraordinaire envahit l'imaginaire de Louise de Savoie, Journal, in op. cit. supra n. 13, t. V, p. 89 : « ... je commencay a predire, par celeste prevision, que mon fils seroit une fois en grand affaire contre les Suisses; car, ainsi que j'estois apres souper en mon bois à Romorantin, entre sept et huit heures, une terrible impression celeste, ayant figure de comete, s'apparut en ciel, vers occident : et je feus la premiere de ma compagnie qui m'en apperceus; mais ce ne fut sans avoir grande peur; car je mescriai si hault que ma voix se pouvoit estendre, et ne disois autre chose sinon : Suisses! Les Suisses! Les Suisses. " 
l'espaule ou en l'estomac ». Le « désordre » du combat ne lui fait pas peur, il est guerrier parmi les autres guerriers, réduisant face à la mort omniprésente la distance qui dans la durée normale le sépare de ses hommes dans une épreuve physique ${ }^{23}$.

Mais c'est surtout dans les heures qui précèdent l'engagement de Cérisoles que montent en surface toutes les données constitutives de la relation triple et indissociée qui unit Celui qui a triomphé de la Mort pour la Rédemption des hommes, celui qui continue ce triomphe en une action de sacrifice continué et une noblesse qui est le corps actif du royaume. Montluc réclame d'avoir été à l'origine de la décision de livrer bataille, grâce à un discours tenu devant un " roy soldat » et son conseil, dans lequel il évoque la "charge " donnée par Dieu à François I $^{\text {er }}$ et la manière mémorable dont celui-ci l'a exercée : « vous avez autant cherché la fortune de la guerre que roy qui jamais ayt esté en France, sans avoir espargné vostre personne non plus que le moindre gentilhomme." L'accomplissement de la royauté sacrale tient ici dans la reconnaissance d'une fusion entre le roi et son peuple, une identité d'acte qui relève d'une tension de sacrifice, du même mépris de la mort qui caractérisa Charles VII à Fornoue. La force française est à nouveau définie en termes d'irrésistibilité, tous les assistants étant « résolus » de mourir ou de vaincre avec leur roi présent parmi eux : "j'oseroy dire que, si nous avions tous un bras lié, il ne seroit encores en la puissance de l'armée ennemie de nous tuer de tout un jour, sans perte de la plus grand part de leurs gens et des meilleurs hommes... " ${ }^{24}$.

Cette profession de foi sacrificielle ne suffit pas. En dernier ressort, dans une invocation qui témoigne d'une intimité intense, c'est vers Dieu que François $I^{\text {er }}$ se tourne solidairement parce que c'est à Dieu qu'il revient d'obéir, parce que c'est pour lui et par lui que l'on doit combattre :

« Alors le Roy leva les yeux vers le ciel, et, joignant les mains, jettant le bonet sur la table, dict: "Mon Dieu, je te supplie qu'il te plaise me donner aujourd huy le conseil de ce que je dois faire pour la conservation de mon royaume, et que le tout soit à ton honneur et à ta gloire". "

La bataille résulte d'un conseil de Dieu, d'une relation de l'homme à Dieu qui procède d'une humilité première : « le roi, après avoir demeuré

23. Les mémoires de messire Martin du Bellay contenant le discours de plusieurs choses advenues au royaume de France, depuis l'an 1513 jusques au trespas du roy François $F$...., in op. cit. supra n. 13, t. V, p. 125-126. Cf. Joumal d'un bourgeois de Paris sous le règne de François premier (1515-1534), Paris, L. Lalanne éd., 1834, p. 22 : «... tellement que le Roy et l'armée furent vingt huict heures à cheval, la lance au poing, armez et sans manger. 》

24. B. de Montuuc, op. cit. supra n. 8 , p. 143. 
quelque peu, se tourna vers moy, disant comme en s'escriant : “Qu'ils combatent! Qu'ils combatent" " ${ }^{25}$.

Et même les défaites, dans un retournement de sens, prennent la forme de signes de Dieu, de marques d'amour de Dieu pour un souverain qui n'eut jamais son pareil parce que souffrant pour Lui, presque souffrant comme son Fils a souffert. À Pavie, en effet, François ${ }^{\text {er }}$ porte une cotte d'armes de toile d'argent soulignant dans le jeu des couleurs chevaleresques la pureté d'âme et l'élection royale de celui qui s'en recouvre ${ }^{26}$, l'humilité et l'innocence d'un être qui ne connaît pas la peur parce qu'il s'avance protégé par Celui qui est apparu aux Apôtres vêtu d'un habillement plus blanc que neige ${ }^{27}$. Mais une fois que ses lansquenets ont été vaincus et que ses Suisses se sont retirés, c'est sur lui que « tout le fais de la bataille tomba ». Son cheval est tué, et lui-même est blessé à la jambe. Autour de lui les morts s'accumulent, de l'amiral Bonnivet à Louis de La Trémouille $^{28}$. À travers une lutte qui le mène « jusques à l'extremité de sa force " et surtout son visage tout maculé de sang et ses blessures au bras et à la main, il rejoint dans la honte même d'être vaincu Celui qui s'est sacrifié pour la Rédemption de l'humanité et dont il portait la livrée désormais déchirée et moquée par les soldats. Il ne se rend que lorsqu'il saisit qu'il ne peut plus résister à la volonté de Dieu.

Mais au cour même de l'événement jaillit le miracle, qui transforme la défaite en victoire, assurant à tous, comme jamais peut-être ne fut, la prégnance de Dieu au corps sacré du roi de France et consacré par le sang qui s'en écoule dramatiquement. La mort qu'il a absolument recherchée n'a pas voulu de lui :

« il se trouva aussi avoir receu quelques harquebuzades dans sa cuyrasse ; mais il avoit pendue au col une croix d'or en forme d'un tres riche joyaux : au dedans y avoit enchassé du boys de la vraye croix, qui en retint les coups, qui furent veus visiblement par ceux qui estoient presens [...] Il voulut apres estre pensé faire son oraison dans la Grande Chartreuse, où estant dans l'Église, il y vist un petit escriteau d'un vers de psalme de David, qui dict : " c'est bien reison, Seigneur, que tu m'ayes abaissé, afin que je puisse desormais mieux recognoistre et craindre ta justice ". Cela luy toucha le cœur ${ }^{29}$.

25. Ibid., p. 147.

26. Sur ce point, Michel de La CuRne de SAINTE-Palaye, Mémoires sur l'ancienne chevalerie considérée comme un établissement politique et militaire, A Paris, $1759,8^{\circ} \mathrm{L} 19$, t. I, p. 123.

27. J'utilise l'analyse de Marc Vulson sieur de LA Colombière, La Science héroïque traitant de la noblesse, et de l'origine des armes... A Paris, Chez Sébastien Mabre-Cramoisy, 1669 , V. 5228 , p. 32-35. La signification spirituelle du blanc est aussi référée à ce qu'il " vient plustost de l'air; ainsi toutes les choses aériennes se revêtent de la couleur blanche, comme la neige. Le cerveau aussi est blanc pour ce qu'il est aéré... ».

28. M. Du Bellay, op. cit. supra n. 23, p. 195-197.

29. P. de Bourdeille, seigneur de Brantome, op. cit. supra n. 11, p. 215-217. 
Il est dans sa souffrance allé au-delà de l'Histoire, happé dans une fraction de temps d'éternité; et si la défaite de Pavie ne détermine pas une crise du pouvoir, c'est peut-être parce que le combat du roi, dans sa dimension sacrificielle et malgré le jugement de Dieu, a été une épreuve de mort et que cette épreuve a souligné la transcendance de la royauté.

La violence et le sang qui s'est épanché du corps royal sanctionnent une intronisation continuée du roi. Il faut qu'il ait été homme comme tous les hommes soumis à l'événement total de la mort pour qu'il soit pleinement roi de Dieu et qu'il se révèle le dominateur de la mort. Le sacré se confirme dans une façon de dissolution temporaire qui est la condition même de son exaltation puisque objectivant une reconnaissance christique du souverain. La Majestas du roi est autant un concept qu'une pratique dépassant la seule défense du pays et des sujets, « pour le salut desquels il est tenu d'exposer sa propre vie $"{ }^{30}$.

Certes le roi est sacré, mais la guerre est l'occasion d'amplifier ou de redoubler la sacralité à travers une relativisation instantanée de l'identité individuelle du monarque. Il faut que l'extraordinaire du pouvoir temporel soit en quelque sorte nié par celui qui l'exerce dans une séquence où le roi n'est plus qu'un parmi tous, agressé et annihilé dans la violence, qu'il passe par une mort à lui-même pour qu'il s'exhausse en tant que manifestation transcendante. Au bout de la violence, il y a l'assurance d'une maîtrise de la mort, qui certifie la vérité ontologique du pouvoir en tant qu'un des centres dynamiques de l'univers. Dans la bataille, il n'y a d'ordre que l'ordre de la guerre et de la mort, dans lequel sont comme absentes les distinctions sociales et politiques, et c'est ce sacrifice de soi qu'accepte le roi qui, à l'issue de la violence, prouve sa sacralité. À la limite, par-delà la polarisation de la violence sur le roi combattant, il y a balancement entre un auto-régicide rituel ${ }^{31}$, humanisation absolue puisque celui qui ne meurt jamais fait tout pour rencontrer la mort, et une surhumanisation, puisque le Prince combattant triomphe de toutes les agressions dirigées à la fois contre lui et contre les siens. Le Mystère de la dignité monarchique est le mystère de la mort acceptée et vaincue, preuve de ce que le « pouvoir des roys est conduit par le vouloir de dieu », Celui dont précisément la « gloire » est « en celant et occultant la mort » ${ }^{32}$.

30. Guillaume DU BELLAY, Instruction sur le faict de la Guerre, extraictes des livres de Polybe, Frontin, Vegece, Cornazan, Machiavelle et plusieurs autres bons autheurs, A Paris, De l'imprimerie de Michel de Vascosan, 1553 (1 ${ }^{\text {re }}$ éd., 1548), p. 5.

31. Sur ce point, Alfred Adler, La Mort est le masque du roi. La royauté sacrée des Moundang du Tchad, Paris, Payot, 1982.

32. Guillaume BuDE, Le Livre de l'institution du Prince, au Roy de France tres chrestien Francoys premier de ce nom, A Paris, Chez Jehan Foucher, 1547, p. 62-65. 
N'est-il pas alors logique que la représentation sublimée du roi renaissant soit celle qui l'identifie à l'archange saint Michel, faisant de ce glaive qu'il tient l'épée de Dieu et de sa violence la violence de Dieu, dans la mesure où la force qui anime la pulsion royale de violence est la force triomphante de la Croix, irrésistiblement donnée lors du rituel du sacre $^{33}$ ?

La Majestas est puissance, et donc participation de la Puissance, elle ne trouve son sens alors que dans sa quête paroxystique d'elle-même qu'est la bataille, quand le souverain et son peuple, unis sacrificiellement l'un à l'autre dans le désir de mort, communient dans un don de grâces que l'acte seul du roi rend toutefois possible. Un des théoriciens de la guerre du $X{ }^{e}$ siècle l'a écrit, dépassant le seuil traditionnel des justifications de la guerre juste, la guerre est plaisir de Dieu, parce qu'elle vise à restaurer l'ordre premier de la Création déformé et altéré par le péché humain, par les divisions et les antagonismes qui règnent parmi les hommes, elle est une remontée au commencement pur, vers le mystère de la Toute-puissance ${ }^{34}$, et ainsi elle se révèle approche et recherche d'éternité.

Si alors c'est avec les rois guerriers du premier $x v^{e}$ siècle que le rituel des obsèques royales subit une inflexion capitale, n'est-ce pas en fonction de cet imaginaire structurant? R. E. Giesey a en effet remarqué que c'est avec la mort de Charles VIII qu'est inaugurée l'opposition corps-effigie, " qui succédait à leur interchangeabilité ». La dépouille royale, traditionnellement portée en terre revêtue ou accompagnée des habits royaux et des insignes de souveraineté, se trouve inhumée nue. C'est l'effigie qui, habillée du costume, porte le sceptre et la main de justice et est exposée séparément du corps pour personnifier triomphalement la Majestas ${ }^{35}$. Le roi mort paraît ainsi vivant, selon $R$. E. Giesey exprimant la " permanence de la dignitas royale survivant à la mort de la personne physique du roi jusqu'à la mise en terre ". Mais peut-être le symbolisme des funérailles rejoint-il essentiellement le symbolisme des batailles royales? Ne décrit-il pas la sacralité comme une figure de triomphe sur la mort à travers l'effigie, image d'immortalité individuelle du souverain s'identifiant à l'éternité de son royaume et donc de son peuple, image d'un combat victorieux qui ne doit cesser jamais?

Peut-être est-ce aussi dans le cadre de cette structure de représentation qui fait la mort intimité du roi, que Henri II, une fois la paix publiée, par-

33. Sur cet aspect, cf. A. DUPRONT, art. cit. supra n. 1, p. 328-329.

34. Michel d'Amboise, Le Guidon des gens de guerre, A Paris, en la boutique de Galliot du Pré [1543], Paris, 1878, p. 151-154.

35. Ralph E. GIESEY, Le Roi ne meurt jamais. Les obsèques royales dans la France de la Renaissance, Paris, Flammarion, 1987, p. 175 et 287. 
ticipe au tournoi fatal de la rue Saint-Antoine, ayant fait dès le 22 mai proclamer que lui-même, en compagnie d'Alphonse de Ferrare, de François de Lorraine et de Jacques de Nemours, lutterait " contre tous venans " en un champ clos? Plusieurs prétextes étaient officiellement donnés. Tout d'abord, la violence chevaleresque était une représentation destinée à célébrer festivement l'union d'Elisabeth de France et de Philippe II d'Espagne et les fiançailles de Marguerite de France et de Philibert Emmanuel de Savoie. Dieu, par sa sainte grâce, a donné enfin la paix à la chrétienté, après une longue "guerre cruelle et violente». Le tournoi répondait à un « devoir " collectif « avec toutes demonstrations de joye, plaisirs, et allegresses, de louer, et celebrer un si grand heur... " En second lieu, il devait permettre de montrer l' " utilité " de l'emploi et du maniement des armes en de "vertueux et louables faictz et actes " destinés à « inciter les jeunes a vertu, et recommander la prouesse des experimentez ${ }^{36}$.

Mais, enfin, n'avait-il pas pour objet de maintenir dans la proximité de la mort un souverain qui venait d'un seul coup de briser toute une Histoire de violence et d'approche solidaire de la personne royale et du "peuple " français d'avec la mort ? Ne visait-il pas à représenter la permanence primordiale du désir de mort indissocié de l'exercice de la dignité monarchique, et ce d'autant plus que le souvenir de la tentative régicide de Caboche est encore vif et aussi qu'un imaginaire circule environnant Henri II d'inquiétantes pronostications? Ne cherchait-il pas à restaurer une identité spécifique de la royauté, qui ne lui survit pas tout simplement parce qu'il est pris conscience de ce que le comportement provocatoire du monarque à l'égard de la mort pouvait se retourner contre l'idéologie absolutiste, la fragilisant, voire la détruisant ?

Car il est évident que si la thématique du sacrifice royal va subsister, elle va voir son contenu évoluer, se reconstituer dans une autre dimension insistant désormais sur la symbolique. La mort va ainsi, avec Charles IX et Henri III, s'approcher différemment du Prince.

Elle fait de Charles IX un roi Christ, rongé intérieurement par la violence et les haines de tous ceux qui se sont dressés dans le royaume contre son autorité. Nul souverain n'a été confronté à des guerres plus atroces que lui. Il les a vécues dans une grande déchirure personnelle, et dans ce rapport douloureux à la violence de ses contemporains et à la perversion humaine se découvre une plénitude sacrale. Mais c'est à la fois

36. La Publication des emprises du tournoy qui doibt estre faict à Paris, ville capitale du royaume de France, pour la solennité des tresheureux mariages du Roy Catholique, avec madame Elizabeth, fille aisnée du Roy Treschrestien. Et du Duc de Savoye avec Marguerite de France. Publié au dict lieu par les Heraux darmes de France, A Lyon, Par Benoist Rigaud, 1559, Lb31 97, n.p. 
à distance et dans l'intériorité que la confrontation avec la mort a lieu, une confrontation lente et usante. L'oraison funèbre, composée par JeanBaptiste Bellaud, développe une identification à Hercule, montrant que depuis sa toute petite enfance, Charles IX n'a vécu que dans les épines et travaux et qu'il s'est épuisé physiquement dans un travail de tous les jours qui cherchait à purger le monde "d'une infinité de monstres" ${ }^{37}$.

Une autre forme d'offrande de soi, mystique également, qui a abouti au triomphe de la Croix qu'authentifie le massacre d'août 1572, inaugurant un nouveau siècle d'or. Le sacrifice n'est plus tension d'instants de guerre, certes recherchés et répétés, révélant une vérité de surnature dans l'individualité monarchique, il est la vie même du roi, toute la vie du roi dans la continuité d'un mimétisme christique. Le roi est l'image du Christ de la Passion, assumant en lui-même le Mal du monde et mourant pour le Bien des hommes. Arnaud Sorbin développe cette figuration tragique, en insistant sur la supériorité de Charles IX sur tous ses prédécesseurs : le roi de la Saint-Barthélemy a fait plus qu'aucun de ses ancêtres, plus même que Clovis à qui il revient d'avoir "confirmé " la vraie foi en France, puisqu'il l'a restituée "miraculeusement" alors qu'elle était "presque perdue ». Cette force qui a été dans toute sa vie fut une force donnée par Dieu, de résister aux tentations diaboliques et de les combattre à distance sans jamais fléchir. Fidélité, abnégation, prière.

Mais la lutte a été souffrance martyrologique. Les apparences sont trompeuses; la mort de Charles IX n'a pas été vraiment naturelle. Le roi de la Saint-Barthélemy est décédé tué par la méchanceté de certains de ses sujets qui, « redoublans de jour en jour leur malice, prodition, et toute impiété, ont tellement outré son cueur, que peu à peu la langueur, produite de la tristesse d'être confronté au mal, l'a amené à la mort ${ }^{38}$. C'est-à-dire que son règne n'a été qu'une perpétuelle montée au Calvaire, une perpétuelle relation à la mort. L'absolu souffrant d'une solitude caractérise désormais le désir de mort royale et la lutte qui lui est corrélative, reflet d'une évolution de l'absolutisme. C'est moins l'acte exalté de violence et de rencontre avec la mort que l'acceptation et l'endurance douloureuses de la violence qui sont censées énoncer la sur-nature royale et donc justifier le Prince comme être d'Absolu.

37. Jean-Baptiste BELLAUD, Oraison funebre du trespas du Roy Tres chrestien Charles neusiesme. A la Royne Mere du Roy Regente de France, A Paris, par Frederic Morel, 1574, Lb33 371, p. 12.

38. Arnaud SORBin, Oraison funebre du tres hault, puissant et tres chrestien Roy de France, Charles $I X$, piteux et debonnaire, propugnateur de la foy, et amateur des bons esprits : prononcée en l'église Nostre-Dame en Paris, le XII de Juillet MD.LXXIIII..., A Paris, Chez Guillaume Chaudiere, 1574, Lb33 376, p. 20-26 (" prodition " pour trahison). 
Contrairement à ce que l'on a tendance à penser, Henri III, poursuivant le processus de démilitarisation de la personne royale, ne va faire qu'amplifier cet imaginaire renouvelé du sacrifice royal; ce à travers le théâtre d'une piété pénitente dont il se veut l'unique centre. Le corps royal n'agit plus, ne cherche plus à s'épuiser dans le combat contre des forces humaines, il s'efforce avant tout de se donner à souffrir, de montrer qu'il est sacrificiel, qu'en lui seul se réalise l'œuvre de purification de la société temporelle. Il est le premier corps du royaume, et donc le corps qui doit être exemple pour tous.

Tout part, pour Henri III, de la constatation que le malheur de la France divisée et déchirée par les misères des troubles civils et religieux a pour origine les vices et péchés des humains. Seule une réformation collective pourrait détourner l'ire divine, réformation irréalisable si celui que Dieu a placé à la tête de la société mondaine ne la commence pas en son propre être. Dès 1578, dans une lettre à Amauld du Ferrier, le roi en vient à désirer supporter la pénitence pour tous les vices et iniquités innombrables, "pour le salut et redemption de tant de pauvre et désolé peuple... ${ }^{39}$. D'où la création de l'Archi-congrégation des pénitents de 1'Annonciation Nostre Dame, dans laquelle Henri III, avec ses confrères, s'engageait à s'abandonner à une existence de communion avec les souffrances du Christ. Et les processions pénitentielles, prolongeant une piété privée mortificatrice, n'eurent de sens que de mettre en scène un monarque supportant et prenant en charge dans son corps souffrant les péchés de son peuple. Dans la crise de sa dignitas, le roi absolu devient le lieu christique de la société temporelle, le lieu où la Vie doit triompher de la mort dans une contention d'adoration mimétique de la Passion. Le pouvoir devient le pouvoir sotériologique de lutter contre les forces de mort qui entraînent le châtiment divin sur tout un peuple, et celui qui l'exerce s'intronise sauveur mystique. L'espace et la durée du désir de mort royal ont capitalement changé, peut-être se matérialisant dans l'événement d'un régicide qui est à la fois sanction collective et quête individuelle.

Toutefois, le premier régicide est l'expression d'un échec idéologique. La fascination pour le côtoiement avec la mort a fini, comme en 1559, par produire la mort du roi. D'où, peut-être, la prise de conscience de ce que la mort doit être évacuée de l'idéologie monarchique pour que la royauté puisse redevenir puissance de Dieu. Certes, en apparence, Henri de Navarre renoue avec l'imaginaire du roi combattant physiquement les forces du mal. Mais le système de représentation va plus loin : la violence

39. Cité in Édouard Frémy, Henri III pénitent. Etude sur les rapports de ce prince avec diverses confréries et communautés parisiennes, Paris, 1885, p. 10-12. 
du roi, lorsqu'elle est racontée, a une autre signification que celle, traditionnelle, qui articulait le temps royal de la bataille à la mise en valeur d'une action divine dans l'être du monarque. Le roi se bat contre les ennemis de la France, mais la mort disparaît de son possible, parce qu'il est hors de la mort, hors de son champ menaçant, parce que sa durée ignore toute rencontre avec la mort. Auparavant, la mort permettait d'assurer de la sur-nature du roi, désormais elle n'a plus de statut dans le discours. Henri de Navarre est l'identité du destin, d'une force que rien ne peut entraver et qui doit conduire le royaume vers un temps régénéré de paix et de bonheur; il est donc une figure de l'Éternité se réalisant à travers son action, sa lutte est irrésistible de puissance d'accomplissement, et aucune force ne peut l'empêcher d'arriver à sa plénitude. Il est l'âme du monde, dont la seule existence vivifiante assure du passage de la France et de son peuple vers la vie retrouvée. Sa royauté est absolue, parce que son être est l'Absolu, simultanément Temps et Histoire réconciliés : «le Ciel l'a promis tel à la France »", et il est un "demyDieu ${ }^{41}$.

Lui aussi est Hercule, mais pensé comme l'agent de l'ordre implacable du devenir conditionnant le devenir de tous les Français, le maître du Temps. Il est dit immortel, parce que sa royauté, voulue de toute éternité, ne peut être que divine et donc éternelle dans le travail qu'elle effectue de rapprocher l'ordre humain de l'ordre universel de la Raison. Le système absolutiste comme système de l'obéissance absolue s'explique à partir de cette conceptualisation.

Toute l'œuvre de réédification politique du règne d'Henri IV vise ainsi à certifier que la mort ne se tient pas dans la sphère du roi absolu. Les rois du Xvi siècle cherchaient à faire venir la mort près d'eux, l'idéologie des années 1589-1610 l'éradique du discours politique. Une autre certitude est imposée que "le roi ne meurt jamais ". C'est en fonction de cette obsession que sa mythique discursive et son déroulement événementiel participent d'une certaine logique. La mythique est une mythique d'éternité, ainsi dans l'Entrée royale de 1610, qui théâtralisait une façon d'apothéose du roi vivant : l'univers tout entier était dit promettre «à leurs Majestez une durée et eternité en leur empire... ${ }^{42}$. Au bout du par-

40. Discours d'Estat, où la necessité et les moyens de faire la guerre à l'Espagne mesme sont richement exposez, A Paris, Chez Jamet Mettayer et Pierre l'Huillier, 1605, Lb35 612, p. 11.

41. Jean Godard, L'Oracle ou le chant de Protée. Contenant la Prediction des vaillances et victoires de Henry IV très Chrestien et tres Victorieux Roy de France et de Navarre. Avec les Trophées du mesme Seigneur. Dediez à sa Majesté par I.G.P., A Paris, Chez Jamet Mettayer et Pierre l'Huillier, 1595, Ye. 55639, p. 10-11.

42. Entrée décrite in Registres de délibérations du bureau de la ville de Paris, 1499-1624, Paris, Bonnardot F. éd., 1883-1953, t. 14, p. 473-501. 
cours urbain, les Parisiens pouvaient voir l'Éternité donner un règne sans fin au roi, à la reine et à leurs enfants. La royauté «florissant à jamais n'aura de fin " à travers des enfants royaux dépositaires du "genus immortale " d'Henri IV, qui tel le phénix revit en eux, dans le sang qui coule en eux et qui pour toujours revivra. Et le déroulement événementiel, en l'occurrence la mort du roi, sous le couteau de Ravaillac, prend effectivement et objectivement au piège les nostalgies ligueuses, dans la mesure où il produit sa propre négation. La mort est " œuvre de Dieu ", parce qu'elle confirme l'immortalité royale en se faisant totalement vie aussitôt dans le petit Louis XIII, dans la confirmation d'un système de domination qui est fondé sur la Raison et l'adéquation du monde humain à l'ordonnancement immuable de l'univers. À la limite, la mort du roi - dans une offrande de soi-même à la violence ? - est une fausse mort, elle consacre la royauté comme lieu de divinité. Et c'est peutêtre parce qu'elle est une illusion de mort, qu'elle fit tant parler d'elle. Elle exalte le règne humain comme règne de paix éternelle.

L'histoire donc du passage, par paliers, vers le second absolutisme, apparaît ici caractérisée par la mise de la personne royale hors du champ de la Mort. Le souverain n'est plus que désir d'obéissance, il n'est plus désir de mort. Il a abandonné la faculté qui était inhérente à sa Majesté de se trouver soudainement propulsé dans l'Autre, dans l'Extraordinaire d'une manifestation divine. Une nouvelle sacralité surgit de cet éloignement, qui toutefois brise un imaginaire de la sur-nature royale, désormais pensée comme rationalité - et justement, rationnellement dans un système de conscience fondé sur le mouvement perpétuel de l'univers, le nouveau roi est roi immédiatement, avant même les funérailles de son père et le Lit de justice qui précède ces dernières, rejoignant, « petit phœnix ", aussitôt la surhumanité paternelle. En devenant éternelle, la royauté proclamait son autonomisation par rapport à l'autre monde.

L'humanisation du Sacré, en la quotidienneté vivante d'un roi Dieu du Xvil siècle, ne fut-elle pas sans déterminer paradoxalement, dans le conscient ou l'inconscient du politique et dans son moyen ou long terme, une déperdition de puissance sacrale? En délaissant ou refoulant son désir premier de mort, la Majesté royale gagnait certes un autre absolu de puissance, mais peut-être perdit-elle un immense absolu d'imaginaire?

Denis Crouzet, Université de Lyon-Jean-Moulin. 